\section{The Contursi Family 20 Years Later: Intrafamilial Phenotypic Variability of the SNCA p.A53T Mutation}

The SNCA/alpha-synuclein p.A53T mutation segregating in the Contursi kindred was the first mutation ever described in inherited Parkinson's disease (PD). ${ }^{1,2}$ We directly examined 10 subjects (4 PD patients and 6 asymptomatic relatives) of a branch of the Contursi kindred. We also collected data on 11 members affected by history, through interviews and inspection of available medical records. Nine had died in the fourth to seventh decade diagnosed with PD (plus psychiatric features in 3), whereas 2 were reported to have severe psychiatric disturbances and were unavailable for examination.

After obtaining ethics approval and written informed consent, all subjects underwent a complete neurological examination (see Video 1) and SNCA p.A53T mutation testing. The $4 \mathrm{PD}$ patients (age, $42 \pm 9.8$ years), and 2 unaffected individuals (age, 29.5 \pm 2.1 years) resulted as heterozygous for the mutation. Two affected (III:1, IV:1) and 2 unaffected (IV:2, IV:6) carriers also underwent extensive neuropsychological and psychiatric assessment, olfaction evaluation, and dopamine transporter imaging with single-photon emission computed tomography (DAT-SPECT); 3T brain MRI was performed in 3 subjects (III:1, IV:2, and IV:6; Fig. 1).

Although the overall phenotype in the 4 patients was typical of SNCA-related PD with early, asymmetric onset, initial good response to dopaminergic therapy and early motor complications, ${ }^{3}$ a relevant variability was observed in the combination and severity of motor symptoms (partially responding to levodopa and DBS in III-9) and nonmotor features (Table 1). Age at onset varied from 26 to 48 years (mean, $32.7 \pm 10.5$ ), with longer disease duration than previously reported (from 2 to 19 years; mean, 9.2 \pm 8.5 ). Cognitive deficits were present in all 4

${ }^{*}$ Correspondence to: Dr. Alfonso Fasano, University of Toronto Movement Disorders Center, Toronto Western Hospital, 399 Bathurst Street, 7 Mc412, Toronto, ON, Canada M5T 2S8;

E-mail: alfonso.fasano@uhn.ca or alfonso.fasano@gmail.com

Funding agencies: This work was partly supported from the Italian Ministry of Health (Ricerca Corrente 2015) and the European Community (FP7-MeFoPa grant).

Relevant conflicts of interest/financial disclosures: Nothing to report. Full financial disclosures and author roles may be found in the online version of this article.

Received: 18 July 2015; Revised: 25 November 2015; Accepted: 6 December 2015

Published online 22 January 2016 in Wiley Online Library (wileyonlinelibrary.com). DOI: 10.1002/mds.26549 patients, ranging from frank dementia in those with longer disease duration (III-8, III-9) to moderate cognitive impairment (III-1) or slight isolated executive dysfunction (IV-1) in those with a shorter one. Depression and anxiety were detected in 3 patients, behavioral disorders in 2, and dysautonomia in 2 . Olfaction was impaired in both tested patients. Although the variable severity could depend on the different disease duration, the wide range of ages at onset among carriers of the same genetic mutation remains unexplained, suggesting the existence of yet unknown environmental, genetic, and/or epigenetic modifiers. 4

Interestingly, 2 asymptomatic carriers, ages 31 and 28 years, were clinically unaffected. Their general cognitive functions were normal and they did not refer any sleep or mood disorder. However, the younger one (IV:2) presented an objective olfactory deficit and an abnormal DAT scan, suggesting an underlying, still subclinical, neurodegenerative process. The existence of SNCA mutation carriers who remain clinically asymptomatic at ages beyond the expected age of onset has been reported, implying reduced penetrance. ${ }^{5}$ However, our carriers were still younger than the latest age of onset in the family; therefore, any conclusion would be merely speculative and further clinical follow-up is necessary.

In conclusion, our data further highlight the relevant intrafamilial phenotypic variability in carriers of the SNCA p.A53T mutation, suggesting a role for yet unknown genetic or environmental modifiers. Follow-up studies are encouraged to better define the clinical spectrum of PD caused by SNCA gene mutations.

Lucia Ricciardi, MD, PhD, ${ }^{1}$ Simona Petrucci, MD, ${ }^{2,3}$ Daniela Di Giuda, MD, ${ }^{4}$ Laura Serra, $\mathrm{PhD},{ }^{5}$

Barbara Spanò, MD, PhD, ${ }^{5}$ Mariachiara Sensi, MD, ${ }^{6}$

Monia Ginevrino, BSc, ${ }^{3}$ Fabrizio Cocciolillo, MD, ${ }^{4}$

Marco Bozzali, MD, PhD, ${ }^{5}$ Enza Maria Valente, MD, PhD, ${ }^{3,7}$ and Alfonso Fasano, $\mathrm{MD}, \mathrm{PhD}^{8, *}$

${ }^{1}$ Sobell Department of Motor Neuroscience and Movement Disorders, Institute of Neurology, University College London, London, United Kingdom; ${ }^{2}$ Department of Neurology and Psychiatry, "Sapienza" University of Rome, Rome, Italy; ${ }^{3}$ IRCCS Casa Sollievo della Sofferenza, CSS-Mendel Institute and San Giovanni Rotondo, Rome, Italy; ${ }^{4}$ Institute of Nuclear Medicine, Università Cattolica del Sacro Cuore, A. Gemelli University Hospital, Rome, Italy; ${ }^{5}$ Neuroimaging Laboratory, IRCCS Santa Lucia Foundation, Rome, Italy; ${ }^{6}$ Department of

Neuroscience-Rehabilitation, S. Anna University-Hospital of Ferrara, Ferrara, Italy; ${ }^{7}$ Department of Medicine and Surgery, University of Salerno, Salerno, Italy; ${ }^{8}$ Morton and Gloria Shulman Movement Disorders Center and the Edmond J. Safra Program in Parkinson's Disease, Toronto Western Hospital, University Health Network, Toronto, Ontario, Canada 

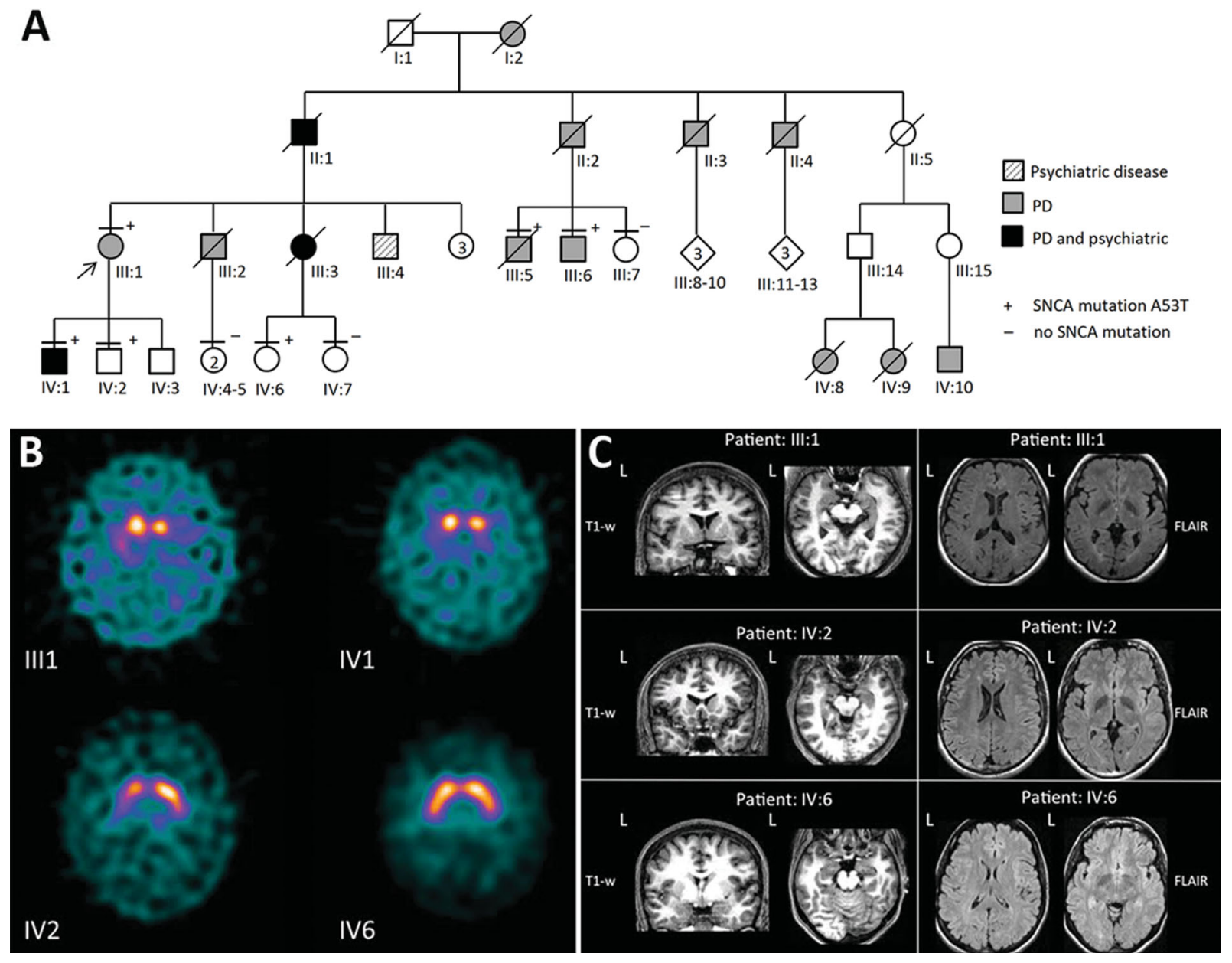

FIG. 1. (A) Simplified pedigree of the family. Black symbols denote affected individuals with both PD and psychiatric disease; gray symbols denote patients with PD; white and gray striped symbols denote patients with psychiatric disease only. A thin horizontal line above symbols denotes clinically and genetically examined individuals. Dead members are marked with a diagonal bar. The arrow indicates the proband. $-=$ no SNCA mutation; + = heterozygous for SNCA p.A53T mutation. (B) Transaxial ${ }^{123}$ I-FP-CIT SPECT slices (1 pixel thick) at the level of the striatum SPECT was abnormal in 2 patients and 1 healthy carrier and normal in subject IV:6. Patients III-1 and IV-1 showed decreased radiotracer uptake bilaterally in the striatum. The asymptomatic carrier, IV-2, also showed a reduction in ${ }^{123}$ I-FP-CIT uptake in the right putamen. Activity was intense in the caudate nuclei and putamina of subject IV-6 (Supporting Table 1). (C) Illustrated here are some images obtained from patients' MR scanning. T1-weighted images (left side) did not reveal any sign suggestive for regional brain atrophy. Fluid-attenuated inversion recovery scans did not show any remarkable macroscopic abnormalities in patients' white matter. [Color figure can be viewed in the online issue, which is available at wileyonlinelibrary.com.]

\section{References}

1. Golbe LI, Di Iorio G, Sanges G, et al. Clinical genetic analysis of Parkinson's disease in the Contursi kindred. Ann Neurol 1996;40: 767-775

2. Polymeropoulos M, Lavedan C, Leroy E et al. Mutation in the alpha-synuclein gene identified in families with Parkinson's disease. Science 1997;276:2045-2047.

3. Kasten M, Klein C. The many faces of alpha-synuclein mutations. Mov Disord 2013;28:697-701.

4. Cooper DN, Krawczak M, Polychronakos C, Tyler-Smith C, Kehrer-Sawatzki $\mathrm{H}$. Where genotype is not predictive of phenotype: towards an understanding of the molecular basis of reduced pene- trance in human inherited disease. Hum Genet 2013;132:10771130

5. Papadimitriou A, Veletza V, Hadjigeorgiou GM, Patrikiou A, Hirano M. Anastasopoulos I. Mutated a-synuclein gene in two Greek kindreds with familial PD: incomplete penetrance? Neurology 1999; $52: 651$.

\section{Supporting Data}

Additional Supporting Information may be found in the online version of this article 